\title{
APROXIMANDO COMUNIDADES DE PRÁTICA E ANÁLISE DE REDES SOCIAIS:
} UMA MOLDURA ANALÍTICA

\section{BRINGING CLOSER COMMUNITIES OF PRACTICE AND SOCIAL NETWORK ANALYSIS: AN ANALYTICAL FRAMEWORK}

\section{APROXIMANDO COMUNIDADES DE PRÁCTICA Y ANÁLISIS DE REDES SOCIALES: UN MARCO ANALÍTICO}

\section{Patricia Kinast De Camillis}

Doutora em Administração pela Universidade Federal do Rio Grande do Sul (UFRGS)

Professora na Universidade do Vale do Rio dos Sinos (Unisinos)

Endereço: Av. Dr. Nilo Peçanha, n. 1600, Bairro Boa Vista, CEP: 91330-002. Porto Alegre, RS, Brasil Telefone: (51) 35911122

E-mail: patriciadecamillis@gmail.com

\section{Débora Junge Baum}

Mestra em Gestão e Negócios pela Unisinos

Professora na Universidade do Vale do Rio dos Sinos (Unisinos)

Endereço: Av. Dr. Nilo Peçanha, n. 1600, Bairro Boa Vista, CEP: 91330-002. Porto Alegre, RS, Brasil Telefone: (51) 35911122

E-mail: deborabaum@gmail.com

\section{Jorge Renato de Souza Verschoore Filho}

Pós-Doutorado em Administração pela Universidade da Califórnia em Berkeley

Professor e Pesquisador na Universidade do Vale do Rio dos Sinos (Unisinos)

Endereço: Av. Dr. Nilo Peçanha, n. 1600, Bairro Boa Vista, CEP: 91330-002. Porto Alegre, RS, Brasil Telefone: (51) 35911122

E-mail: jorgevf@unisinos.br

Artigo recebido em 20/10/2019. Revisado por pares em 26/11/2019. Reformulado em 30/11/2019. Recomendado para publicação em 15/12/2019. Publicado em 30/04/2020. Avaliado pelo Sistema double blind review.

CCopyright 2020 UNISUL-PPGA/Revista Eletrônica de Estratégia \& Negócios. Todos os direitos reservados. Permitida citação parcial, desde que identificada a fonte. Proibida a reprodução total. Revisão gramatical, ortográfica e ABNT de responsabilidade dos autores. 


\section{RESUMO}

Em um contexto organizacional no qual a Gestão do Conhecimento tem recebido crescente valorização, as Comunidades de Prática surgem como ferramenta gerencial para promover a aprendizagem organizacional, que pode ser vista como um processo inerentemente social. Grande parte dos fatores críticos de desenvolvimento de Comunidades de Prática Virtuais estão ligados à sua estrutura social. Sendo assim, o objetivo final é apresentar uma proposta teórica para a análise das Comunidades de Prática sob o viés das Redes Sociais. A análise feita dos construtos apresentados possibilita destacar as relações informais existentes em Comunidades de Prática, mesmo quando intencionalmente criadas.

Palavras-chaves: Comunidades de Prática Virtual; Análise de Rede Social; Gestão do Conhecimento; Desempenho Organizaciona; Relações Informais.

\section{ABSTRACT}

In an organizational context in which Knowledge Management has received increasing appreciation, the Communities of Practice appear as a managerial tool to promote organizational learning, which can be seen as an inherently social process. Most of the critical development factors of Communities of Practice are linked to their social structure. Therefore, the final objective is to present a theoretical proposal for the analysis of Communities of Practice under the bias of Social Networks. The analysis of the presented constructs makes it possible to highlight the informal relations existing in Communities of Practice, even when intentionally created.

Keywords: Virtual Communities of Practice; Social Network Analysis; Knowledge Management; Organizational Performance; Informal Relations.

\section{RESUMEN}

En contexto organizacional en el cual la Gestión del Conocimiento ha recibido creciente valorización, las Comunidades de Práctica surgen como herramienta gerencial para promover el aprendizaje organizacional, que puede ser visto como un proceso inherentemente social. Gran parte de los factores críticos de desarrollo de Comunidades de Práctica están ligados a su estructura social. Siendo así, el objetivo final es presentar una propuesta teórica para el análisis de las Comunidades de Práctica bajo el sesgo de las Redes Sociales. El análisis hecho de los constructos presentados posibilita destacar las relaciones informales existentes en Comunidades de Práctica, aun cuando intencionalmente creadas.

Palabras Clave: Comunidades de Práctica Virtual; Análisis De Red Social; Gestión Del Conocimiento. Desempeño Organizacional; Relaciones Informales. 


\section{INTRODUÇÃO}

A gestão do conhecimento tem recebido crescente valorização por parte das organizações, à medida que percebem que a habilidade em capitalizar conhecimento pode ser uma fonte de vantagem competitiva (LEE; SUH; HONG, 2010). Nesse contexto, surgem as comunidades de prática, como uma ferramenta para promover o compartilhamento e a criação de conhecimento a partir da troca de experiências entre seus membros. As comunidades de prática criam valor para as organizações à medida que possibilitam a resolução de problemas de forma rápida e efetiva, contribuem para a capacitação e o desenvolvimento de seus membros, alavancam o compartilhamento de conhecimento tácito e disseminam novos conhecimentos. Além disso, facilitam a geração de ideias, uma vez que promovem reflexões coletivas sobre problemáticas específicas da organização, reunindo esforços e diferentes experiências na busca de soluções. (WENGER; McDERMOTT; SNYDER, 2002).

Por proporcionarem esses benefícios, as comunidades de prática passaram a ser intencionalmente implementadas em diferentes organizações (POWERS, 2004; LEE; SUH; HONG, 2010; SCARSO; BOLISANI, 2008). O desenvolvimento de novas tecnologias e o desafio de trabalhar com organizações grandes e dispersas resultaram na criação de comunidades de práticas virtuais como solução para manter as interações, mesmo considerando as barreiras geográficas (DUBÉ, BOURHIS; JACOB, 2006).

A concepção das comunidades de prática está baseada nos conceitos de aprendizagem social e aprendizagem situada, que consideram a aprendizagem como um processo de participação e interação social (WENGER, 1998). Nesse sentido, uma vez que a aprendizagem é vista como um processo inerentemente social e que a sua organização em comunidade traz justamente a força da relação social como facilitador para o aprendizado, uma série de fatores críticos de desenvolvimento de comunidades de prática estão ligados à sua estrutura social e à forma como seus membros se relacionam. Desta forma, se torna adequado analisar as comunidades de prática sob o viés das redes sociais.

$\mathrm{Na}$ visão das ciências sociais, "redes (sociais) são uma forma de pensar sobre sistemas sociais que focam nossa atenção nas relações entre as entidades que compõem o sistema" (BORGATTI; EVERETT; JOHNSON, 2013, p. 1-2). A análise das comunidades de 
prática sob o viés das redes sociais se torna relevante, à medida que a forma como a rede está estruturada impacta nos resultados de determinado grupo de trabalho ou organização (CUMMING; CROSS, 2003). A análise de rede social proporciona uma melhor compreensão de uma determinada rede, pois mostra como seus membros se relacionam, o quão densa a rede é, quem são as pessoas centrais e as periféricas, dentre outros fatores (BORGATTI; EVERETT; JOHNSON, 2013).

No entanto, os pontos a serem analisados e as possíveis oportunidades de atuação para o desenvolvimento de comunidades de prática não estão plenamente identificados, uma vez que existem, na literatura, poucos estudos aplicando a análise de redes sociais em comunidades de prática de organizações; e os que o fazem, utilizam apenas a metodologia de Análise de Rede Social para identificar, caracterizar e analisar a rede social de uma Comunidade de Prática, a exemplo da pesquisa realizada por Kremer, Akahoshi e Cavaleiro (2017) em uma rede formada pelos membros de uma cooperativa.

Sendo assim, o artigo irá elencar as características de estrutura da rede social que impactam no desenvolvimento de comunidades de prática; também se propõe a identificar os construtos que caracterizam a estrutura de rede social para comunidades de prática; apresentando, por fim, uma proposta teórica para a análise das comunidades de prática sob o viés das redes sociais, integrando as abordagens.

Ao propor tais objetivos, além de contribuir para realização de estudos futuros que usem os construtos propostos que alinham Comunidades de Prática e Análise de Redes Sociais, o artigo aponta que, aproximando essas duas abordagens, é possível identificar características que estão na origem do conceito de Comunidades de Prática, que foram "deixadas de lado" no tratamento gerencial, e que indicam pontos nos quais a gerência terá atuação limitada, que são: as relações informais que se constituem em ambientes virtuais. 0 artigo está estruturado da seguinte forma: revisão do conceito de Comunidade de Prática desde seu surgimento até a utilização de viés gerencial; os fatores críticos para o desenvolvimento das Comunidade de Práticas nas organizações; Análise de Rede Social; apresentação da proposta de construtos; e a conclusão.

\section{COMUNIDADES DE PRÁTICA VIRTUAIS: DA APRENDIZAGEM SITUADA AO GERENCIAMENTO}


Existem, na literatura, diferentes perspectivas sobre aprendizagem, cada qual enfatizando diferentes aspectos do fenômeno. A aprendizagem social é uma delas, cujo foco principal é a aprendizagem como participação social (WENGER, 1998). Segundo Bandura (1971), no sistema de aprendizagem social, padrões de comportamento são adquiridos através de experiências diretas ou pela observação do comportamento de outros. O autor reforça que aprender por experiência é uma das formas mais rudimentares de aprendizado, pois todas as pessoas são confrontadas diariamente com situações novas, com as quais precisam lidar e resolver.

A teoria de aprendizagem social, caracterizada pela participação social, é apresentada por Wenger (1998) com uma divisão em quatro componentes ou formas de aprendizado, que são: significado, na qual o aprendizado ocorre como uma experiência learning as experience; prática, no qual o aprendizado ocorre fazendo - learning as doing; comunidade, na qual o aprendizado ocorre por pertencimento - learning as belonging; e, também, identidade, na qual o aprendizado ocorre ao tornar-se algo/alguém no contexto de uma comunidade - learning as becoming.

Wenger (2000) reforça que, em um sistema de aprendizagem social, competências são definidas histórica e socialmente, ou seja, pessoas adquirem essas competências em suas comunidades sociais, através de suas experiências. Sendo assim, a troca de experiências entre pessoas com diferentes competências caracteriza a aprendizagem social. A partir da aprendizagem social, Lave e Wenger (1991) propuseram o termo Aprendizagem Situada, como sendo mais complexa do que o termo Aprender Fazendo. Nesse sentido, a aprendizagem emerge de interações sociais através da participação no mundo social, onde aprendizagem não é uma atividade específica, mas um aspecto de todas as atividades (WENGER, 1998; LIMA, 2013).

O desenvolvimento de novas tecnologias e de ferramentas que proporcionam maior conexão entre as pessoas facilita a aprendizagem social. Nesse contexto, Bingham e Conner (2011) propõem o Novo Social Learning, onde as mídias sociais impulsionam a interação entre as pessoas (de todos os níveis, de cada parte da organização e de qualquer canto do globo) e oferecem oportunidades de aprendizado. Além disso, "o novo social learning foca no compartilhamento da informação, colaboração e cocriação, e exige que a noção de 
treinamento seja ampliada" (BINGHAM; CONNER, 2011, p. 19), isto é, ir além dos treinamentos formais. Desta forma, a Aprendizagem Situada ganha novos contextos.

Os conceitos de aprendizagem social e situada deram origem ao conceito de comunidades de prática, cuja implementação foi impulsionada com o advento das tecnologias de informação e comunicação atuais. O termo Comunidades de Prática foi utilizado inicialmente nos estudos da administração por Lave e Wenger (1991), baseado na teoria de aprendizagem situada e tendo como foco as interações entre profissionais mais experientes e novatos, e o progresso dos indivíduos de uma posição periférica para um status de especialista (GUDBERG; MACKNESST, 2009; Ll et al, 2009).

A abordagem destaca a aprendizagem através da interação social situada e informal, ao invés de um processo mecânico e planejado de transmissão cognitiva. Tal interação faz com que o aprendizado esteja relacionado com o que realmente precisa ser conhecido a respeito das complexidades da prática real, isto é, no contexto no qual se está inserido. Desta forma, a participação periférica e o envolvimento ativo na prática são chaves para o aprendizado (COX, 2005).

De acordo com Lave e Wenger (1991, p. 98), Comunidade de Prática é “um sistema de relacionamento entre pessoas, atividades e o mundo; desenvolvido com o tempo e em relação a outras comunidades que tangenciam ou se sobrepõem" como uma condição intrínseca de existência do conhecimento. Entende-se também que o grupo não é unificado, pois os envolvidos possuem diferentes interesses e pontos de vista (COX, 2005).

Outros pesquisadores desenvolveram estudos que aprofundaram a discussão teórica da Comunidade de Prática, abordando diferentes aspectos. Brown e Duguid (1991), por exemplo, mostraram como grupos informais se formam para improvisar soluções a problemas quando o conhecimento gerencial não dá conta, trazendo, então, o aspecto da “inovação, de criar um novo conhecimento" e não apenas "replicar" práticas. (COX, 2005)

Posteriormente, Wenger (1998) utilizou-se de aspectos teóricos da sociologia e da educação, para construir uma teoria de aprendizagem social, na qual o foco se volta para a socialização e a aprendizagem, e o desenvolvimento da identidade dos indivíduos (LI et al., 2009). Comunidade de Prática passa, então, a ser considerada como um grupo de pessoas 
com engajamento mútuo - estabelecendo normas e relacionamentos - e um repertório comum - linguagem, rotinas, artefatos e estórias -, que aprendem em conjunto em uma determinada organização - ou, pelo menos com um senso de empreendimento conjunto (WENGER, 1998).

Segundo Wenger (1998), nas comunidades de prática, o significado é negociado através do processo de participação e reificação, isto é, dando forma a experiência pela produção de objetos, que podem ser abstrações, ferramentas, símbolos, histórias, termos e conceitos. Porém, a existência de uma comunidade de prática pode não ser evidente aos seus próprios membros, o que destaca sua informalidade. Comunidades de prática, portanto, não seriam entidades estáticas e estáveis, pois envolvem, ao longo do tempo, novos membros, enquanto outros saem. Além disso, considerando a definição original de Lave e Wenger (1991), a gestão não conseguiria estabelecer uma Comunidade de Prática, apenas facilitar que emerjam espontaneamente e dar suporte ao seu desenvolvimento.

Assim, um aspecto importante das comunidades de prática é a relação entre formalidade e informalidade. Para Lave e Wenger (1991), a comunidade de prática pode ocorrer em um ambiente de sistema formal de aprendizagem, embora entenda o aprender como algo informal - não estruturado e não planejado. Na publicação posterior de Wenger (1998) a respeito do tema, o autor também considera a informalidade como aspecto relevante, pois entende que o engajamento autêntico se dá para além da formalidade e pode haver formatos e propósitos não esperados por parte do designer do sistema formal.

\subsection{COMUNIDADE DE PRÁTICA PELA VERTENTE GERENCIAL}

Em um trabalho mais recente, Wenger, McDermott e Snyder (2002) trouxeram o conceito de Comunidade de Prática para a esfera gerencial, definindo-a como "um grupo de pessoas que compartilham uma preocupação, um conjunto de problemas, ou a paixão sobre algum tema, e que aprofundam seu conhecimento e especialidade nessa área, interagindo de forma contínua" (WENGER; McDERMOTT; SNYDER, 2002, p. 4). Segundo os autores, uma comunidade de prática é uma combinação de três elementos fundamentais: 
a) Domínio: define o tema, conjunto de questões ou assunto comum aos participantes. Um domínio de conhecimento bem definido inspira os membros a participarem, guia sua aprendizagem, além de dar identidade ao grupo.

b) Comunidade: cria a estrutura social que facilita o aprendizado. Uma comunidade forte estabelece interações e relações entre os membros, baseadas em respeito mútuo e confiança.

c) Prática: conjunto de ferramentas, informações, procedimentos, ideias, entre outros, a respeito do domínio. A prática é o conhecimento específico que a comunidade desenvolve, compartilha e mantêm.

Contando que tenham esses três elementos fundamentais, as comunidades de prática podem assumir diferentes formas, variando quanto ao seu tamanho, duração, alocação (local ou distribuída) e composição (homogêneas ou heterogêneas). Além disso, suas fronteiras podem permanecer dentro de uma organização, entre unidades de negócio ou até entre diferentes organizações (WENGER; McDERMOTT; SNYDER, 2002).

Em tempo de empresas globais e redes de comunicação disponíveis e conectadas, as comunidades de prática chamadas "distribuídas" tornaram-se mais frequentes. Porém, em função da distância, tamanho e até de diferenças culturais, Wenger, McDermott e Snyder (2002) apontam que as comunidades distribuídas enfrentam algumas dificuldades. Uma delas diz respeito ao desafio de construir relacionamentos pessoais e de confiança. Segundo os autores, uma vez que os membros têm menos contato uns com os outros, existem poucas oportunidades para conversas particulares, não permitindo a construção de uma relação mais próxima com outros participantes. Com isso, ideias ou solicitações de ajuda são feitas somente pelo website da comunidade virtual e nem sempre são atendidas. Fatores culturais também se tornam uma barreira na construção do relacionamento nas comunidades.

Como não podem contar com encontros face-a-face como principal veículo de interação, as comunidades distribuídas precisam encontrar outros meios para compartilhar e trocar conhecimento. Consequentemente, as comunidades virtuais ganham mais espaço, como uma solução de interação para pessoas geograficamente dispersas (GUDBERG; MACKNESST, 2009; WENGER; MCDERMOTT; SNYDER, 2002). Entende-se por comunidades de 
prática virtuais aquelas que utilizam ferramentas tecnológicas de comunicação e informação como seu principal meio de interação, sem necessariamente excluir encontros presenciais (DUBÉ; BOURHIS; JACOB, 2006).

Embora o conceito original de comunidades de prática aponte que elas emergem de forma natural e espontânea, Wenger, McDermott e Snyder (2002) sugerem que organizações podem construí-las e cultivá-las, como mecanismo para aumentar a competitividade (LI et al., 2009). Seguindo essa linha, organizações investiram recursos, intencionalmente, criando comunidades de prática em diferentes setores (YAMKLIN; IGEL, 2012; SOUZA-SILVA, 2009; LI et al, 2009; GUDBERG; MACKNESST, 2009; GAZZOLI, 2012; HAFEEZ; ALGHATAS, 2007; LEE; SUH; HONG, 2010; WENGER, 2000). Empresas globais, como IBM, 3M, Xerox, Dell, Shell, Accenture e Caterpillar, inclusive consideram comunidades de prática como estratégias em sua cadeia de valor de conhecimento (POWERS, 2004; LEE; SUH; HONG, 2010; SCARSO; BOLISANI, 2008).

As comunidades de prática criam valo, através da resolução de problemas de forma rápida e da promoção e transferência de melhores práticas, ampliando o desenvolvimento de habilidades dos membros, trazendo benefícios como desenvolvimento profissional, apoio à solução de problemas, economia de tempo, sinergia entre unidades e novas estratégias competitivas (FERNANDES, CARDOSO, CAPAVERDE, SILVA, 2016). Além disso, facilitam a geração de ideias para novos produtos e serviços, contribuindo para os resultados de longo prazo da organização (YAMKLIN; IGEL, 2012).

Para Wenger e Snyder (2000), as comunidades de prática podem impulsionar a estratégia da empresa, iniciar uma nova linha de negócio, promover e espalhar melhores práticas e até ajudar no recrutamento e retenção de talentos, uma vez que essas pessoas enxergam nas comunidades de prática uma possibilidade de desenvolvimento, aprendizagem e contato com conhecimentos de ponta em determinado tema. Adicionalmente, Lee, Suh e Hong (2010) consideram-nas como ferramentas de gestão do conhecimento que promovem melhorias como aumento de eficiência, promoção de capacidade de resposta e introdução de inovações.

Outro ponto importante de criação de valor refere-se à natureza dos conhecimentos abordados nas comunidades de prática, pois possibilitam alavancar o conhecimento tácito, 
através de interações entre as pessoas e engajamento em busca de avanços em suas práticas (McDERMOTT, 2000). Considera-se também que o contexto de uma comunidade de prática promove oportunidades para que indivíduos relatem histórias e experiências, compartilhando, assim, o conhecimento tácito (HAFEEZ e ALGHATAS, 2007).

Além disso, "por possuírem práticas semelhantes, quando os membros de uma comunidade de prática discutem sobre seus problemas cotidianos, eles colaboram reflexivamente até o ponto em que inventam soluções inovadoras" (SOUZA-SILVA, 2009, p. 178). Em resumo, o valor de uma comunidade de prática está na sua habilidade em ajudar a organização a atingir suas metas, agindo de forma efetiva na resolução de problemas e compartilhando conhecimento fora das barreiras de suas estruturas formais (McDERMOTT, 2000; LESSER; STORCK, 2001).

\subsection{FATORES CRÍTICOS PARA O DESENVOLVIMENTO DE COMUNIDADES DE PRÁTICA}

Apesar de não existir, na literatura, um consenso sobre quais são os fatores críticos para o desenvolvimento de comunidades de prática, uma série de estudos buscaram identificar os mesmos de forma pontual, como a compreensão dos elementos que promovem a participação dos membros (GUDBERG; MACKNESST, 2009), a importância da ligação com a estratégia, através de metas tangíveis (YAMKLIN; IGEL, 2012), o papel da cultura organizacional (SOUZA-SILVA, 2009) e a implicação da escolha das tecnologias que suportam as comunidades (WENGER; WHITE; SMITH, 2009). Outros autores apresentam fatores críticos de forma mais ampla, explorando os principais pontos que promovem o desenvolvimento de comunidades de prática e agrupando-os em grandes pilares, temas, fases ou dimensões (LEE; SUH; HONG, 2010; McDERMOTT, 2000; SCARSO; BOLISANI; SALVADOR, 2009; SCHIMITT; SCHIMITT, 2017; WENGER; McDERMOTT; SNYDER, 2002).

Cabe ressaltar que o próprio conceito de comunidades de prática surgiu com base em teorias de aprendizado social e que sua organização em comunidade traz justamente a força da estrutura social como facilitadora para o aprendizado. Assim, uma série de fatores críticos de desenvolvimento estão ligados justamente à estrutura da comunidade de prática e à forma como seus membros se relacionam. Para Wenger, McDermott e Snyder (2002, p. 58), "o coração da comunidade é a rede de relacionamentos entre seus membros." 
Considerando que, de forma geral, o propósito de uma comunidade de prática é o desenvolvimento de seus membros, através da construção e troca de conhecimento (WENGER; SNYDER, 2000), o alcance desse objetivo depende, obrigatoriamente, da interação entre os membros. É justamente por meio das contribuições dos mesmos nos ambientes da comunidade (virtuais ou físicos) que essa troca de conhecimento acontece. Cowan e Menchaca (2014) reforçam que, desde o conceito inicial de comunidade de prática formulado por Wenger e Lave (1991), quando observaram aprendizes no trabalho, o aprendizado não ocorria de forma passiva, mas pelo desenvolvimento de conhecimento através de trocas sociais. No contexto das comunidades de prática, aprendizes são ativamente envolvidos em um processo dinâmico, onde o conhecimento é formado, não pela simples aquisição de informações, mas tornando-se informado em um contexto onde o aprendizado é negociado através de interações (WENGER; McDERMOTT; SNYDER, 2002; COWAN; MENCHACA, 2014).

Cross et al. (2001) reforçam que a criação e o compartilhamento de conhecimento ocorrem mais frequentemente através da interação entre pessoas, e não pela troca de documentos ou consultas em base de dados. Em especial, a resolução de problemas complexos em curto espaço de tempo exige reflexão e profundidade de análise, o que pode ser acelerado com a participação de mais pessoas. Sendo assim, relações "continuam a ser críticas na transferência de conhecimento e difusão de inovações e ideias" (CROSS et al., 2006, p. 32). Além disso, em seu estudo sobre aprendizado organizacional, Pahor, Skerlajav e Dimovsky (2008) verificaram que é mais provável que o aprendizado ocorra em grupos que possuem relações mais densas, ou seja, com maior nível de interação.

A interação entre os membros de comunidades de prática pode ocorrer através de diferentes canais. Enquanto encontros presenciais, como workshops ou conferências, proporcionam interação face-a-face (WENGER; McDERMOTT; SNYDER, 2002), a tecnologia vem possibilitando cada vez mais opções de ferramentas, como videoconferências (DUBÉ; BOURHIS; JACOB, 2006). Comunidades virtuais comumente possuem seus próprios espaços na web, com ambientes virtuais que proporcionam ferramentas como fóruns, blogs, biblioteca e canal para perguntas e respostas (WENGER; WHITE; SMITH, 2009). 
Uma das questões estruturais destacadas na literatura, e que difere comunidades de prática de outras estruturas formais, é a participação voluntária dos membros. Enquanto nas organizações, colaboradores são designados a comporem times de projeto ou grupos de trabalho, nas comunidades de prática esses colaboradores participam se desejarem. Nesse contexto, os membros podem optar por aderir ou não à comunidade de prática e por ter uma participação mais ou menos ativa, ou seja, contribuindo mais ou menos nos ambientes da comunidade. Sendo assim, um fator crítico de desenvolvimento para a comunidade de prática é a habilidade de gerar entusiasmo, relevância e valor, não só para atrair, mas também para engajar seus membros (WENGER; McDERMOTT; SNYDER, 2002). Logo, o nível de envolvimento dos membros impacta diretamente no volume de contribuições e, consequentemente, no volume de trocas, compartilhamento e criação de conhecimento.

Os motivos que levam pessoas a participarem de uma comunidade de prática podem variar. Alguns o fazem porque se importam com o domínio da comunidade e querem ver seu desenvolvimento, enquanto outros encontram valor na própria comunidade, pois conseguem interagir com seus colegas ou pessoas que compartilham o mesmo interesse e irão valorizar contribuições sobre o tema. Outros, ainda, consideram o aprendizado promovido na comunidade de prática como motivador (WENGER; McDERMOTT; SNYDER, 2002).

Buscando aprofundar a questão crítica da participação voluntária e do nível de engajamento dos membros, Gudberg e Macknesst (2009) realizaram estudo no qual identificam os fatores que facilitam ou dificultam a participação de membros em uma comunidade de prática virtual. Destacaram cinco fatores que influenciam na participação, sendo: emoção (sentimento de cada participante em relação à experiência), tecnologia (diferentes tecnologias aplicadas e a familiaridade dos participantes com as mesmas), conectividade (sentimentos de pertencimento ou não à comunidade), entendimento das normas (regras e ritmo de aprendizagem) e tensões de aprendizagem (como a tensão entre novatos versus experientes ou teoria versus prática).

De igual forma, Gatti (2012) buscou relacionar fatores facilitadores e inibidores no uso de comunidades de prática. Os fatores foram divididos em aspectos humanos, tecnológicos e organizacionais, cada qual com subitens. No estudo foram considerados 
fatores facilitadores: o alinhamento das comunidades de prática à estratégia e objetivos da empresa, a avaliação e medição e desempenho das comunidades e os processos de comunicação organizacional. Foram identificados como fatores inibidores: a cultura organizacional, a falta de tempo dos membros para participarem, a tecnologia disponível, além de questões comportamentais, como a resistência em compartilhar conhecimento. Segundo McDermott (2000) e Wenger, McDermott e Snyder (2002), incentivar a comunidade de prática com eventos, atividades ou discussões cria um constante movimento e manter esse ritmo também é importante para a manutenção da participação. Adicionalmente, a promoção de espaços comunitários, sejam virtuais ou eventos presenciais, como conferências ou workshops, possibilita que os membros se conheçam, criem vínculos e se sintam entusiasmados e engajados.

A motivação é um dos fatores principais para o engajamento em atividades de aprendizado e compartilhamento de conhecimento (KIESLINGER; PATA; FABIAN, 2009). Shirky (2011) explora diferentes movimentos de participação colaborativa e as motivações por trás dos mesmos. Além de motivações pessoais, Shirky (2011) cita motivações sociais, como o desejo de participação e de compartilhamento. O autor também afirma que as motivações sociais reforçam as motivações pessoais, se ampliando mutuamente, numa malha de retroalimentação. Enquanto as novas redes de comunicação encorajam a participação em comunidades e o compartilhamento, isso também fornece apoio para autonomia e competência.

Apesar do sucesso das comunidades de prática depender da interação e envolvimento de seus membros, diferentes níveis de participação são esperados. "Uma vez que as pessoas têm diferentes níveis de interesse na comunidade, a expectativa de participação igualitária é irreal" (WENGER; McDERMOTT; SNYDER, 2002, p. 55). Usualmente, são encontrados nas comunidades de prática três níveis de participação: um pequeno grupo principal, que participa mais ativamente das discussões, eventos, e identifica novos tópicos; um grupo ativo, que participa regularmente das atividades da comunidade, mas com menor intensidade de contribuição e, por fim, um grupo periférico, que caracteriza-se pela maior porção dos membros, com rara participação. Ser membro periférico não significa que não está aprendendo ou se desenvolvendo na comunidade. Além disso, os níveis de participação não são fixos e, portanto, os membros podem transitar entre eles (WENGER, 1998; 
WENGER; McDERMOTT; SNYDER, 2002). Para McDermott (2000), mais importante do que balancear os níveis de participação, é contar com um grupo principal envolvido e ativo, pois são essas pessoas que mais influenciam os demais e promovem a comunidade. Por outro lado, Cross et al. (2006) alertam que, caso o grupo principal seja composto por poucas pessoas, a comunidade de prática pode ser tornar vulnerável à saída de algum desses membros.

Uma forma de reforçar a participação dos membros do grupo principal e de reconhecer esse envolvimento diferenciado é através da atribuição de papéis formais. A existência de papéis formais em comunidades de prática é considerada um fator crítico de desenvolvimento relacionado à estrutura e ao relacionamento entre os membros. Segundo Scarso e Bolisani (2008, p. 380), “uma comunidade não é autossustentável e requer um número de papéis de suporte formalmente definidos." Dependendo da forma como a comunidade de prática se organiza, demandas surgem, como a liberação de novos membros, a organização do conteúdo gerado, a promoção de eventos, entre outros.

Esses papéis podem variar de acordo com o estágio de maturidade de cada comunidade, ou da forma como seus membros se adaptam (WENGER; McDERMOTT; SNYDER, 2002). Papéis claramente designados tornam-se crescentemente importantes, à medida que as comunidades de prática aumentam em tamanho e importância. Os papéis formais podem levar a um maior envolvimento e responsabilidade dos membros, e ajudam as pessoas a legitimar o tempo gasto trabalhando nas comunidades de prática (DUBÉ; BOURHIS; JACOB, 2006).

Dubé, Bourhis e Jacob (2005) afirmam que o papel de liderança é um ingrediente essencial para o sucesso das comunidades de prática. Essa liderança não necessariamente precisa ser hierárquica e, em muitos casos, o líder é escolhido pelos próprios membros, refletindo reconhecimento, respeito e um alto nível de conhecimento sobre o domínio. Sua função pode incluir tarefas como: manter o foco no domínio, promover relacionamento entre os membros, identificar prioridades ou temas atuais e encorajar uma cultura de participação (LEE; SUH; HONG, 2010; SCARSO; BOLISANI, 2008;) e ainda promover eventos, workshops, espaços ou momentos onde os membros possam se conhecer e trocar informações (WENGER; McDERMOTT; SNYDER, 2002). Em algumas comunidades de prática, 
as atividades mencionadas também podem ser feitas por um moderador ou facilitador, deixando para a liderança apenas a gestão macro na comunidade.

Outros papéis ou funções de suporte também podem existir, como o de especialista no domínio ou gestor do conhecimento. Ao receber o papel de especialista, essa pessoa é considerada referência no domínio da comunidade, possuindo tipos específicos de informação ou conhecimento técnico (CROSS; PRUSAK, 2002; SCARSO; BOLISANI, 2008). Membros especialistas podem contribuir na resolução de questões complexas, temas atuais ou auxiliando membros iniciantes no seu desenvolvimento na determinada prática. Já o papel de gestor do conhecimento é o de coordenar o volume de conhecimento gerado na comunidade de prática.

Independentemente dos papéis, o perfil dos membros da comunidade de prática impacta no conteúdo gerado, na profundidade das discussões e na probabilidade de interação. Cada comunidade pode ter um perfil de membros mais homogêneo ou heterogêneo, dependendo das características e do histórico de seus participantes. Segundo Wenger, McDermott e Snyder (2002), uma diversidade de culturas nacionais pode acarretar problemas de comunicação, tendo a própria língua como um fator inibidor. Além disso, os traços culturais de cada nacionalidade refletem padrões de comportamento e de postura colaborativa. A cultura organizacional, por sua vez, influencia, caso os membros da comunidade de prática sejam originados de diferentes organizações, cada qual com seus processos, normas e prioridades. Já a cultura profissional reflete padrões de cada profissão, como a linguagem, vocabulário específico, normas, valores e até a forma de se vestir (WENGER; McDERMOTT; SNYDER, 2002; DUBÉ, BOURHIS; JACOB, 2006).

Percebe-se, portanto, que perfis de membros heterogêneos dificultam a interação entre os membros. Segundo Dubé, Bourhis e Jacob (2006), pessoas tendem a interpretar informações baseadas nos seus filtros culturais, o que traz um potencial para distorções ou erros de interpretação. Cross, Borgatti e Parker (2002) destacam que pessoas tendem a se comunicar mais com aqueles com quem têm perfil similar ao seu. Esse achado foi confirmado no estudo de Pahor, Skerlajav e Dimovsky (2008), envolvendo aprendizado em organizações. 
Por outro lado, comunidades com perfis mais homogêneos, compostas por membros com a mesma formação ou funções, tendem a replicar soluções similares. Segundo Cross et al. (2006), quando as comunidades de prática são compostas por membros com perfis muito parecidos, com as mesmas competências ou histórico, suas discussões são influenciadas por paradigmas dominantes e dificilmente vistas sob um novo prisma. Pahor, Skerlajav e Dimovsky (2008) argumentam que sistemas sociais heterogêneos, onde a interação entre pessoas com diferentes trajetórias promove maior exposição a ideias diferentes, são sistemas mais propícios a gerar inovação. Lee, Suh e Hong (2010) reforçam a importância de criar conhecimentos, focando em inovação como forma da comunidade de prática se adaptar ao longo do tempo. Em suma, perfis heterogêneos contribuem para o objetivo da comunidade de prática em construir conhecimento, promovendo inovação. No entanto, isso pode se tornar mais desafiador, cabendo aos líderes compreender o perfil dos membros da comunidade de prática, entender seus impactos e gerenciar os mesmos, seja através da construção de normas, prioridades ou entendimentos comuns (DUBÉ; BOURHIS; JACOB, 2006).

Conforme foi visto, as comunidades de prática são uma forma de promover aprendizagem, na qual um grupo de pessoas com uma prática em comum aprofunda seu conhecimento sobre a mesma, através da troca de experiências, interagindo de forma contínua. As comunidades de prática criam valor para as organizações, à medida que promovem disseminação de melhores práticas, rápida resolução de problemas e criação de novos conhecimentos. Para atingir esse propósito, a interação entre os membros é fundamental, assim como o nível de envolvimento dos mesmos, uma vez que a participação nas comunidades é voluntária e depende de outras motivações. Para tanto, o estabelecimento de papéis formais auxilia na interação e manutenção do envolvimento, assim como na qualificação do conteúdo compartilhado. Por fim, destaca-se que o perfil dos membros impacta nas comunidades, sendo que perfis mais heterogêneos promovem maior inovação.

Nesse contexto, percebe-se que a forma como a comunidade de prática está estruturada e como seus membros se relacionam impacta diretamente no seu desenvolvimento. Por isso se propõe a utilização da perspectiva da Análise de Rede Social (ARS) para enriquecer a compreensão acerca das Comunidades de Prática. Na seção a seguir, 
destacam-se os conceitos abordados em Análise de Redes Sociais que tornam possível a aproximação com Comunidades de Prática.

\section{ANÁLISE DE REDES SOCIAIS}

O termo "redes" está frequentemente presente no contexto contemporâneo. Em sua essência e conceito mais simples, rede é uma teia de elementos e elos entre estes, e possuem três elementos: atores, conexões e a rede como um todo (BARABÁSI, 2009).Os estudos de redes e, em especial, sua utilização nas ciências sociais, trouxeram avanços significativos, produzindo explicações para fenômenos sociais em uma variedade de disciplinas (BORGATTI; EVERETT; JOHNSON, 2013).

A visão de rede para as ciências sociais é explicada por Borgatti, Everett e Johnson (2013, p. 1-2): "redes são uma forma de pensar sobre sistemas sociais que focam nossa atenção nas relações entre as entidades que compõem o sistema." Ou seja, redes sociais têm enfoque, não nas pessoas ou entidades que compõe a rede e seus atributos, mas sim na relação entre essas entidades (OTTE; ROUSSEAU, 2002). Para Freeman (2004), a abordagem das redes sociais está fundamentada na noção intuitiva de que os padrões das conexões sociais das quais os atores estão envolvidos têm consequência para esses atores.

A Análise de Redes Sociais tem suas raízes em diversas perspectivas teóricas, como a psiquiatria, no trabalho de Moreno (1934), a antropologia, na corrente britânica dos anos 40 e a sociologia estrutural (MIZRUCHI, 2006). A sociometria, desenvolvida por Moreno (1934), teve um papel importante, à medida que promoveu um caminho para tornar estruturas sociais abstratas em algo tangível. Nos anos 40 e 50, um estudo desenvolvido pelo Laboratório de Redes do MIT (Massachusetts Instituteof Technology) explorou o impacto de diferentes estruturas de redes de comunicação na velocidade para resolução de problemas, concluindo que estruturas tinham melhor performance, onde a distância entre todos os nós, até o integrador, era a menor. Nos anos 70, o trabalho de Granovetter (1973), na sociologia, teve uma contribuição chave sobre a influência dos laços fracos (BORGATTI et al., 2009).

Uma rede social pode apresentar diferentes estruturas, dependendo de quanto seus atores se conhecem e quais se relacionam. Em um grupo de cinco pessoas, por exemplo, é possível que todos se conheçam e se relacionem, ou que todos tenham uma única pessoa 
conhecida em comum, que as liga com os demais. É consenso entre a literatura do tema de que diferentes estruturas de redes impactam de forma diferente na performance e resultado de determinado grupo, organização ou seus indivíduos (BRASS, 1984; CROSS et al.,2001; CUMMING; CROSS, 2003; SPARROWE et al., 2001).

Esse impacto se dá, uma vez que diferentes estruturas influenciam a forma como a comunicação ocorre, na sua velocidade e no seu acesso. No entanto, não existe uma melhor estrutura ou a que proporcione melhores resultados. Cumming e Cross (2003) verificaram que redes integradas produzem melhores resultados em grupos sem rotina, com trabalho complexo ou que envolva várias expertises. Por outro lado, para grupos com atividades rotineiras, uma estrutura hierárquica pode apresentar melhores resultados. Além disso, uma melhor estrutura para o grupo de trabalho não significa melhor realização para o indivíduo. Quando o indivíduo assume um papel central na estrutura e a comunicação depende dele, sua importância e poder aumentam (BRASS, 1984; CUMMING; CROSS, 2003). Segundo Sparrowe et al. (2001), estruturas de redes sociais aumentam ou restringem o acesso a recursos valiosos. Além disso, redes sociais transmitem identidade e suporte social. Nesse contexto, a posição do indivíduo em uma determinada rede social pode the prover vantagens. Borgatti et al. (2009) reforçam o pressuposto de que a estrutura importa, considerando este um axioma fundamental de redes sociais.

Considerando que a análise das redes sociais resume-se à estrutura e à posição (BORGATTI; EVERETT; JOHNSON, 2013) e que diferentes estruturas impactam de forma diferente (BRASS, 1984; CROSS et al.,2001; CUMMING; CROSS, 2003; SPARROWE et al., 2001), uma forma de investigá-las é utilizar o prisma da centralização. Segundo Butts (2009), a centralização mede o quanto as conexões estão concentradas em um pequeno número de nós. Em outras palavras, Borgatti, Everett e Johnson (2013) consideram que a centralização é uma medida da rede como um todo, que se refere à medida em que a rede é dominada por um único nó.

É possível também medir o grau de centralização de cada um dos atores. Em seu conceito básico, a "centralidade" é uma propriedade da posição de um nó em uma rede, ou seja, reflete a importância estrutural de um nó na rede (BORGATTI; EVERETT; JOHNSON, 2013). Segundo Cowan e Menchaca (2014), a centralidade se refere à quantidade de 
conexões presentes na rede de um ator. Quanto mais conexões, maior seu grau de centralidade. A centralidade é o conceito mais estudado, quando a análise é feita a nível dos nós da rede (BORGATTI et al., 2009) e pode ser medida, considerando diferentes perspectivas, como a de grau (degree), a de intermediação (betweeness) e a de proximidade (closeness) (OTTE; ROUSSEAU, 2002; SCOTT, 2013; BORGATTI; EVERETT; JOHNSON, 2013).

Atores com alta centralidade de grau são por vezes chamados de "conectores centrais" e aqueles com baixa centralidade de grau são chamados de "periféricos". Em seu estudo sobre redes informais em organizações e os papéis críticos para o seu desempenho, Cross e Prusak (2002) destacam o papel do conector central, caracterizando-o como um indivíduo que liga as pessoas umas às outras na rede, uma vez que sabe quem pode fornecer determinada informação crítica ou técnica. Com isso, toda a rede depende do conector central para finalizar tarefas. Para Barabási (2009), os conectores centrais são um componente importante em uma rede social e uma propriedade fundamental na maioria das redes, presentes em diversos sistemas complexos.

Por outro lado, indivíduos com papéis centrais na rede podem, por vezes, se tornar sobrecarregados, e diminuir a velocidade do fluxo de informações. O estudo de Cross et al. (2006) identificou que pessoas com esse perfil acabam investindo muito tempo no compartilhamento de melhores práticas e não se atualizando em sua área de atuação. Além disso, podem ser um ponto de vulnerabilidade, uma vez que a rede depende muito de sua atuação.

Alguns indivíduos são mais centrais em uma rede do que outros, isso se deve ao motivo pelo qual são procurados pelos demais. Borgatti e Cross (2003) indicam que a probabilidade de um indivíduo procurar uma informação de outra pessoa é uma função de percepção entre saber o que o outro sabe, dar valor ao que o outro sabe e ter acesso ao outro. Em um nível coletivo, a estrutura dessas relações e percepções reflete o aprendizado de uma rede e seu potencial para reagir a novos temas e oportunidades. Cross et al. (2001) apresentam qualidades relacionais que promovem compartilhamento de conhecimento em uma organização, dentre as quais, destaca-se: o conhecimento, o acesso, o engajamento e a segurança. Assim, entende-se que o conhecimento é compartilhado quando o indivíduo sabe o que o outro sabe, tem acesso a ele, ambos estão interessados em se envolver com o 
problema e sentem confiança mútua para compartilhar. O benefício dessa interação acontece para ambos, isto é, quem dá assistência para solução de um problema ou disponibiliza seu conhecimento também aprende (SHAH, CROSS, LEVIN, 2018).

Frequentemente encontram-se incorporados à rede grupos de atores que interagem entre si de uma forma que podem ser considerados uma entidade separada. Esses clusters são praticamente uma rede separada dentro de uma rede maior (COWAN; MENCHACA, 2014). Atores que compõem clusters coesos tendem a compartilhar ideias, metas ou normas. Além disso, tendem a ter resultados similares, como comportamentos, atitudes ou práticas (BORGATTI; EVERETT; JOHNSON, 2013). Quando um cluster está muito desconectado do restante dos atores da rede, este é chamado de "silo", e torna-se um grupo isolado. Em um estudo sobre análise de redes sociais em comunidades de prática, Cross et al. (2006) afirmam que os silos dificultam o fluxo de informação e tornam o resultado das comunidades menos efetivo.

Para identificar o motivo desta divisão em clusters, é necessário analisar quais atributos comuns poderiam explicar seus laços mais intensos (BORGATTI; EVERETT; JOHNSON, 2013). Alguns atributos vinculam-se a valores culturais, tema ou tipo de especialidade, locação física ou hierarquia formal (CROSS et al., 2006). Outros fatores comuns para fragmentação de redes identificados por Cross, Borgatti e Parker (2002) em um estudo sobre colaboração em redes informais foram: estilo de liderança hierárquica, dispersão física e trabalho virtual, resistência a soluções "não inventadas aqui", fluxo de processos ou descrição de cargos que não contribuam para a colaboração na rede.

Neste contexto, alguns atores destacam-se na rede, os chamados de brokers, que assumem um papel de intermediadores, mantendo os clusters conectados, porém, sem ter o mesmo número de laços diretos que um conector central (CROSS; BORGATTI; PARKER, 2002; CROSS et al., 2006). A centralidade de intermediação reflete em que medida um nó facilita o fluxo de uma rede (OTTE; ROUSSEAU, 2002; BORGATTI; EVERETT; JOHNSON, 2013). Para Cross et al. (2006), brokers são atores ideais para se trabalhar quando se quer difundir rapidamente certa informação na rede, como uma melhor prática. Borgatti et al. (2009) reforçam o entendimento e alertam que atores com alto grau de centralidade de intermediação possuem poder, à medida que podem acelerar ou diminuir fluxos entre os 
clusters. Assim, a Análise das Redes Sociais contribui com dois conceitos para o estudo das Comunidades de Prática: centralização e clusters. Portanto, na próxima seção, sintetizam-se os principais construtos da ARS nas Comunidades de Prática em uma proposta de moldura analítica.

\section{APRESENTAÇÃO DA MOLDURA ANALÍTICA}

Com base nos fundamentos teóricos apresentados, destacam-se como principais construtos da estrutura de redes sociais para comunidades de prática: interação entre os membros, envolvimento, papéis formais, perfil dos membros, centralização e clusters.

Desta forma, os construtos foram compilados no Quadro 1 (um).

Quadro 1 - Moldura de Análise

\begin{tabular}{|c|c|c|}
\hline Construto & Descrição & Referências \\
\hline $\begin{array}{l}\text { Interação entre } \\
\text { membros }\end{array}$ & $\begin{array}{l}\text { Condição fundamental para que ocorra } \\
\text { o compartilhamento e a construção de } \\
\text { conhecimento na comunidade de } \\
\text { prática. } \\
\text { Caracteriza-se pela relação direta entre } \\
\text { os membros. }\end{array}$ & $\begin{array}{l}\text { Wenger e Snyder (2000) } \\
\text { Wenger; McDermott e Snyder (2002) } \\
\text { Cross et al. (2001; 2006) } \\
\text { Pahor, Skerlajav e Dimovsky (2008) } \\
\text { Cowan e Menchaca (2014) }\end{array}$ \\
\hline Envolvimento & $\begin{array}{l}\text { Nível de participação dos membros, uma } \\
\text { vez que a mesma é voluntária. Deriva de } \\
\text { motivações pessoais intrínsecas ou } \\
\text { extrínsecas e de motivações sociais. } \\
\text { Diferentes níveis de participação são } \\
\text { esperados. }\end{array}$ & $\begin{array}{l}\text { McDermott (2000) } \\
\text { Wenger, McDermott e Snyder (2002) } \\
\text { Gudberg e Macknesst (2009) } \\
\text { Shirky (2011) } \\
\text { Gatti (2012) }\end{array}$ \\
\hline Papéis Formais & $\begin{array}{l}\text { Membros com papéis de suporte } \\
\text { reconhecidos na comunidade de prática } \\
\text { que atuam para aprimorar seu } \\
\text { desempenho. }\end{array}$ & $\begin{array}{l}\text { Wenger, McDermott e Snyder (2002) } \\
\text { Dubé, Bourhis e Jacob (2006) } \\
\text { Scarso e Bolisani (2008) } \\
\text { Lee, Suh e Hong (2010) }\end{array}$ \\
\hline Perfil dos Membros & $\begin{array}{l}\text { Pode se caracterizar por um perfil de } \\
\text { membros mais homogêneo ou } \\
\text { heterogêneo, impactando de forma } \\
\text { diferente na comunicação, interação e } \\
\text { tipo de conhecimento gerado. }\end{array}$ & $\begin{array}{l}\text { Cross, Borgatti e Parker (2002) } \\
\text { Cross et al. (2006) } \\
\text { Wenger, McDermott e Snyder (2002); } \\
\text { Dubé, Bourhis e Jacob (2006) } \\
\text { Pahor, Skerlajav e Dimovsky (2008) } \\
\text { Scarso e Bolisani (2008) }\end{array}$ \\
\hline Centralização & $\begin{array}{l}\text { Demonstra como a centralidade dos } \\
\text { atores está distribuída na rede. } \\
\text { Conectores centrais destacam-se pelo }\end{array}$ & $\begin{array}{l}\text { Cross e Prusak (2002) } \\
\text { Otte e Rousseau (2002) } \\
\text { Barabási (2009) }\end{array}$ \\
\hline
\end{tabular}




\begin{tabular}{|c|c|c|}
\hline Construto & Descrição & Referências \\
\hline & alto grau de influência na rede. & $\begin{array}{l}\text { Butts (2009) } \\
\text { Borgatti et al. (2009) } \\
\text { Borgatti, Everett e Johnson (2013) } \\
\text { Scott (2013) } \\
\text { Cowan e Menchaca (2014) } \\
\text { Shah, Cross e Levin (2018) }\end{array}$ \\
\hline Clusters & $\begin{array}{l}\text { Tendem a ter um comportamento mais } \\
\text { coeso entre si, mas dificultam o fluxo de } \\
\text { comunicação. Atributos dos atores } \\
\text { podem explicar a segmentação. Brokers } \\
\text { destacam-se, intermediando clusters na } \\
\text { rede. }\end{array}$ & $\begin{array}{l}\text { Otte e Rousseau (2002) } \\
\text { Cross, Borgatti e Parker (2002) } \\
\text { Cross et al. (2006) } \\
\text { Borgatti et al. (2009) } \\
\text { Borgatti, Everett e Johnson (2013) } \\
\text { Scott (2013) } \\
\text { Cowan e Menchaca (2014) }\end{array}$ \\
\hline
\end{tabular}

Fonte: Elaborado pelos autores (2020).

Considerando que diversas empresas têm investido em Comunidades de Prática como ferramenta gerencial, entende-se que é importante fornecer abordagens que possam analisar, de forma ampla, o desenvolvimento das Comunidades de Prática nas organizações. Entender a Comunidade de Prática como uma Rede Social é bastante promissor, já que ambos conceitos possuem elementos constitutivos em comum: estruturas e atores que se relacionam entre si, sendo a análise das relações que se estabelecem essencial para compreensão de suas dinâmicas.

Assim, a primeira proposição foi, a partir de diversos estudos a respeito de Comunidades de Prática, organizar uma compilação dos fatores críticos para o desenvolvimento gerencial dessa ferramenta, que são: interação entre os membros, envolvimento, papéis formais e perfil dos membros. A partir disso, foi possível identificar as características que possibilitaram a aproximação com a abordagem Análise de Rede Social, para, então, incluir mais dois itens: centralização e cluster, propondo uma moldura analítica de Estrutura de Rede Social para Comunidades de Prática.

Os dois primeiros construtos, interação e envolvimento, indicam diretamente uma característica essencial tanto em comunidade de prática quanto em redes sociais, que são as relações entre os indivíduos. Os papéis formais contribuem para entendermos o quanto a organização consegue interferir na comunidade de prática, à medida que esse papel é definido pela organização. Cabe, então, verificar o quanto este construto poderá ser relevante para a interação e envolvimento dos membros da Comunidade de Prática. Já a compreensão sobre o perfil dos membros irá contribuir para entendermos se a interação e o 
envolvimento possuem associação com perfis mais homogêneos ou não. O que pode nos dar uma pista quanto a capacidade de inovação de uma determinada comunidade de prática analisada.

A Análise de Rede Social busca compreender a respeito da posição dos atores envolvidos, o que complementa a compilação feita inicialmente a partir do referencial de Comunidades de Prática, ampliando seu entendimento. Segundo Mizruchi (2006), embora a análise de redes sociais aborde uma ampla gama de assuntos, três áreas merecem atenção especial por causa de sua relevância teórica, são elas: os efeitos da centralidade do agente/atores, a natureza das relações entre as organizações e a identificação de subgrupos da rede. Para o objetivo proposto aqui, vinculado ao desenvolvimento de Comunidades de Práticas nas organizações, desconsideramos a análise da "natureza das relações entre as organizações". Assim, centralidade e subgrupos estão incluídos nos construtos de "centralização" e "clusters", respectivamente.

Centralização e cluster contribuem para aprimorar o entendimento dos construtos anteriores: a relação entre o perfil dos membros e formação de clusters; a relação entre os papeis formais e a centralização; o quanto o envolvimento e a interação estão restritos a grupos específicos (clusters ou ao redor de determinados atores). Além disso, centralização e cluster podem apontar o quanto as relações informais, isto é, que estão fora da estrutura formal determinada pela organização - o que é difícil de ser gerenciável - estão presentes em uma Comunidade de Prática Virtual.

Os dois construtos destacados a partir da Análise das Redes Sociais podem apontar para o que, na versão original das Comunidades de Prática, é considerado como sendo algo bastante relevante, mas que, porém, na versão gerencial é deixado de lado, ou minimizado, que são as relações informais.

A aproximação das pessoas pode se dar segundo seus próprios interesses, independentemente do que foi planejado incialmente pela gestão. Assim, o aprendizado se dá em tópicos nos quais os próprios indivíduos, em suas relações cotidianas, na prática, entendem, isto é, dão significado como sendo mais interessante ou útil (para a empresa ou não). 
A informalidade e o não planejamento são características importantes originalmente nas Comunidades de Prática e que, mesmo sendo deixadas de lado na versão gerencial, podem aparecer ao analisarmos a "comunidade" pela ótica da Análise das Redes Sociais através dos construtos da centralização e dos clusters.

Desta forma, características que tendem a serem apontadas pela gestão como problemáticas ou dificultadores e que devem ser minimizados, podem indicar que as pessoas estão realmente assumindo as Comunidades de Prática como local para construção de conhecimentos significativos para si mesmo e para seu contexto de trabalho. Desta forma, a gerência, ao pensar ações que minimizem os "aspectos problemáticos" deve ter um olhar mais cuidadoso quanto a determinadas características.

\section{CONSIDERAÇÕES FINAIS}

Ao destacar os fatores críticos para o desenvolvimento das comunidades de prática, elencar as características de estrutura da rede social que impactam nesse processo e também identificar os construtos que caracterizam a estrutura de rede social para comunidades de prática, foi possível apresentar uma proposta teórica para a análise das comunidades de prática sob o viés das redes sociais. Isso contribui para a organização do campo de estudo que busca a aproximação dessas duas abordagens, pois apresenta uma possibilidade de análise estruturada e comparável entre si.

Ao retomar as discussões iniciais das COPs, podemos identificar que a informalidade é algo característico e que muitas vezes é o que possibilita a aprendizagem daquilo que importa, no ambiente de trabalho, para resolver problemas ou realizar o trabalho de maneira que o grupo diretamente envolvido considera que é o melhor. Assim, o aparecimento de cluster e centralização podem ser encarados não mais como um problema, mas apenas como um aspecto não gerencial do conhecimento organizacional, apesar de todos os esforços da gestão em controlá-los. Desta forma, o estudo contribui em apontar a ferramenta de ARS como capaz de identificar características nas Comunidades de Prática Virtuais, que muitas vezes são desconsideradas ou classificadas como algo problemático: as relações informais. 
Por fim, para operacionalizar o estudo dos construtos propostos, nossa sugestão para estudos futuros aplicados com a temática de Comunidades de Prática é de utilizar a metodologia de ARS, através de uma etapa quantitativa, incluindo auxílio de softwares específicos, com foco nas relações estabelecidas entre os membros, para verificar o grau de destaque de cada um desses construtos.

A ARS é considerada um mecanismo formal para representação, medição e modelagem de estruturas relacionais (BUTTS, 2009). A principal tarefa é demonstrar de forma gráfico-teórica as propriedades que caracterizam estruturas, posições e propriedades da rede. Cross et al. (2001) afirma que a análise de redes sociais é uma forma sistemática e rica de avaliar redes, mapeando e analisando relacionamentos entre pessoas, times, departamentos ou até organizações inteiras. Além disso, sugere-se uma investigação qualitativa, posterior, para aprofundar a compreensão das relações estabelecidas entre os membros, mas também entender se há e quais são as relações que se dão entre os construtos.

Desta forma, buscamos contribuir para compreensão cada vez mais aprofundada sobre as relações entre os diferentes atores dentro de uma organização, que contribuem para geração e disseminação do conhecimento. A partir dessa compreensão, poder propor e desenvolver ações que contribuam de forma efetiva para a gestão do conhecimento organizacional, levando em conta que ainda podem existir aspectos difíceis de coordenar e direcionar, mas que, mesmo assim, são importantes nos processos de aprendizagem que geram o conhecimento.

\section{REFERÊNCIAS}

BANDURA, Albert. Social learning theory. New York: General Learning Press. 1971.

BARABÁSI, Albert-László. Linked (Conectado) - A nova ciência dos networks. São Paulo: Leopardo, 2009.

BINGHAM, Tony. CONNER, Marcia. O novo social learning. São Paulo: Évora, 2011.

BORGATTI, S. P. CROSS, Rob. A relational view of information seeking and learning in social networks. Management Science, v. 49, n. 4, p. 432-445, apr. 2003.

BORGATTI, S. et al. Network analysis in the social sciences. Science Magazine, v. 323, p. 892895, feb. 2009. 
BORGATTI, S. EVERETT, Martin G. JOHNSON, Jeffrey C. Analysing social networks. Londres, Sage, 2013.

BRASS, Daniel. J. Being in the right place: a structural analysis of individual influence in an organization. Administrative Science Quarterly, v. 29, p. 518-539, 1984.

BROWN, John Seely. DUGUID, Paul. Organizational learning and communities of practice: toward a unified view of working, learning and innovation, Organization Science 2(1) (1991) 40-57.

BUTTS, Carter T. Revisiting the foundations of network analysis. Science, v. 325, p. 414-416, jul. 2009.

COX, Andrew. What are communities of practice? Journal of Information Science 2005; 31; 527.

COWAN, John E. MENCHACA, Michael P. Investigating value creation in a community of practice with social network analysis in a hybrid online graduate education program.

Distance Education, v. 35, n. 1, p. 43-74, 2014.

CROSS, R. et al. Knowing what we know: supporting knowledge creation and sharing in social networks. Organizational Dynamics, v. 30, n. 2, p. 100-120, fall 2001.

CROSS, R. et al. Using social network analysis to improve communities of practice. California Management Review, v. 49, n. 1, p. 32-60, fall 2006.

CROSS, R., BORGATTI, S. P. PARKER, A. Making invisible work visible: using social network analysis to support strategic collaboration. Management Review, California, v. 44, n. 2, p. 25-46, winter 2002.

CROSS, R., PRUSAK, Laurence. The people who make organizations go - or stop. Harvard Business Review. R0206G, jun. 2002.

CROSS, R., et al. Managing collaboration: improving team effectiveness through a network perspective. California Management Review, v. 50, n. 4, p. 74-98, summer 2008.

CUMMING, Jonathon. N. CROSS, Rob. Structural properties of work groups and their consequences for performance. Social Networks, v. 25, p.197-210, 2003.

DUBÉ, Line. BOURHIS, Anne. JACOB, Réal. The success of virtual communities of practice: the leadership factor. The Eletronic Journal of Knowledge Management, v. 3, issue 1, p. 23-34, 2005.

DUBÉ, Line; BOURHIS, Anne; JACOB, Réal. Towards a typology of virtual communities of practice. Interdisciplinary Journal of Information, Knowledge and Management, v. 1, p. 6993, 2006.

FERNANDES, F. Roberta., CARDOSO, Tiago. Alves., CAPAVERDE, Lisiane. Zynger; Silva, Helena. Fátima. Nunes. Comunidades de prática: uma revisão bibliográfica sistemática sobre casos 
de aplicação organizacional. AtoZ: novas práticas em informação e conhecimento, 5(1), 44 52. 2016.

FREEMAN, Lynton. The development of social network analysis - a study in the sociology of science. Vancouver, BC, Empirical Press. Canada, 2004.

GATTI, Patricia V. B. Fatores facilitadores e inibidores no uso das comunidades de prática: um estudo de caso em uma empresa alemã. Dissertação (mestrado). Universidade Estadual Paulista. Faculdade de Engenharia, Bauru, 2012.

GAZZOLI, Patricia. Comunidades de prática enquanto viabilizadoras de projetos comuns em ambientes turbulentos: uma abordagem crítica. RAC, Rio de Janeiro, v. 16, n. 6, art. 3, p. 806-826, nov./dez. 2012.

GRANOVETTER, Mark. The strenght of weak ties. American Journal of Sociology, v. 78, n. 6, may, 1973.

GUDBERG Karen. MACKNESST J. Foundations of communities of practice: enablers and barriers to participation. Journal of Computer Assisted Learning, v. 25, p. 528-538, 2009.

HAFEEZ, Khalid. ALGHATAS Fathalla. Knowledge management in virtual community of practice using discourse analysis. The Electronic Journal of Knowledge Management, v. 5 Issue 1, p. 29-42, 2007.

KIESLINGER, Barbara. PATA, Kai. FABIAN, Claudia Magdalena. A participatory design approach for the support of collaborative learning and knowledge building in networked organizations. iJac, v. 2, issue 3, p. 34-38, ago. 2009.

KREMER, Andreia Maria, AKAHOSHI, Wesley. Batista, CAVALHEIRO, Rafael Todescato. A Comunidade De Prática Sob A Ótica Da Análise De Redes Sociais: Uma Aplicação Em Uma Cooperativa Brasileira. Perspectivas Online: Humanas \& Sociais Aplicadas, v.7, n.20, p.2842, 2017.

LAVE, Jean , WENGER, Etienne. Situated learning: legitimate peripheral participation in communities of practice. Cambridge University Press: Cambridge, 1991.

LEE Jeehae., SUH Eui-ho, HONG Jongyi. A maturity model based CoP evaluation framework: a case study of strategic CoPs in a Korean company. Expert Systems with Applications, v. 37, p. 2670-2681, 2010.

LESSER, Eric. L. STORCK, John. Communities of practice and organizational performance. IBM Systems Journal, v. 40, n. 4, p. 831-841, 2001.

LI, Linda. et al. Evolution of Wenger's concept of community of practice. Implementation Science. March, 2009.

LIMA, José Jerônimo de Menezes. Efeitos de comunidades de prática na capacidade absortiva em empresas intensivas em conhecimento. Tese (doutorado). Universidade do Vale do Rio dos Sinos, Programa de Pós-Graduação em Administração, São Leopoldo, RS, 2013. 
McDERMOTT, Richard. Knowing in community: 10 critical success factors in building communities of practice. IHRIM Journal, march, 2000.

MIZRUCHI, M. S. Análise de redes sociais: avanços recentes e controvérsias atuais. Revista de Administração de Empresas, v. 46, n. 3, p. 72-86, jul./set. 2006.

MORENO, Jacob L. Who shall survive? a new approach to the problem of human interrelations. Nervous and Mental Disease Publishing Co. Washington, D.C. 1934

OTTE, Evelien; ROUSSEAU, Ronal. Social Network Analysis: a powerful strategy, also for the information sciences. Journal of Information Science, v. 28, n. 6, 2002.

PAHOR, Marko, SKERLAVAJ, Miha, DIMOVSKI, Vlado. Evidence for the network perspective on organizational learning. Journal of the American Society for information Science and Technology, v. 59, n. 12, p. 1984-1994, out. 2008.

POWERS, Vicki. Virtual communities at caterpillar foster knowledge sharing. American Society for Training and Development, p. 40-45, jun. 2004.

SCARSO, Enrico. BOLISANI, Ettori. Communities of practice as structures for managing knowledge in networked corporations. Journal of Manufacturing Technology Management, v. 19, n. 3, p. 374-390, 2008.

$\therefore$; _ _ SALVADOR, Luigi. A systematic framework for analyzing the critical success factors of communities of practice. Journal of Knowledge Management, v. 13, n. 6, p. 431447, 2009.

SCHIMITT, Sabrina Rebelo; SCHIMITT, Rafael de Araújo Rios. Comunidades de Prática: fatores críticos de sucesso à manutenção e suas dimensões de análise. RAD Vol.19, n.1, Jan/Fev/Mar/Abr, p.32-63, 2017.

SCOTT, John. Social network analysis. Third Edition. Sage Publications. 2013.

SHAH, Neha Parikh; CROSS, Rob; LEVIN, Daniel. Z. Performance Benefits From Providing Assistance in Networks: relationships that generate learning. Journal of Management, 44(2), 412-444. 2018

SHIRKY, Clay. A cultura da participação. Rio de Janeiro: Zahar, 2011.

SOUZA-SILVA, Jader. Condições e desafios ao surgimento de comunidades de prática em organizações. RAE, v. 49, n. 2, p. 176-189, abr./jun. 2009.

SPARROWE, Raymond. T. et al. Social networks and the performance of individuals and groups. Academy of Management Journal, v. 44, n. 2, p. 316-325, 2001.

WENGER, Etienne. Communities of practice. Learning, meaning and identity. Cambridge: University Press, 1998.

WENGER, Etienne. Communities of practice and social learning systems. Organization articles, v. 7, n. 2, p. 225-246, 2000. 
WENGER, Etienne; MCDERMOTT Richard, SNYDER William. Cultivating communities of practice: a guide to managing knowledge. Harvard Business School Press: 2002.

WENGER, Etienne; SNYDER William. Communities of practice: the organizational frontier. Harvard Business Review, p. 139-145, jan./fev. 2000.

WENGER, Etienne; WHITE, Nancy, SMITH, John. D. Digital habitats: stewarding technology for communities. CPsquare: Portland, OR, 2009.

YAMKLIN, Sophon, IGEL Barbara. Communities of practice purposefully designed for improving business performance. Knowledge and Process Management, v. 19, n. 4, p. 189202, 2012. 